\title{
К проблеме выбора коррекционных компьютерных программ для восстановления когнитивных функций у пациентов кардиологического профиля
}

\author{
Антон В. Солодухин ${ }^{1,2^{*}}$, Михаил С. Яницкий ${ }^{2}$, Андрей В. Серый² \\ 1 ФГБНУ «Научно-исследовательский институт комплексных проблем сердечно-сосудистых заболе- \\ ваний», г. Кемерово, Российская Федерация \\ 2 ФГБОУ ВО «Кемеровский государственный университет», г. Кемерово, Российская Федерация \\ *E-mail: mein11@mail.ru \\ ORCID ID: http://orcid.org/0000-0001-8046-5470, http://orcid.org/0000-0003-3049-8594, http://orcid. \\ org/0000-0002-9318-4333
}

\begin{abstract}
Аннотация
Введение. Целью обзорной статьи стало изучение используемых в психокоррекционной практике компьютерных программ, направленных на восстановление когнитивных орункций у пациентов карлиологического профриля. В настоящее время наблюдается высокая распространенность нарушений когнитивной сореры среАи Аиц пожи^ого возраста в российской популяции. В том числе снижение когнитивного функционирования в силу влияния заболевания возникает у пациентов карАиологического профиля, что приводит к проблемам социальной алаптации и снижает их качество жизни. В связи с этим требуется изучение существующих компьютерных программ когнитивной реабилитации Аля пациентов карАиологического проориля.

Теоретическое обоснование. По результатам теоретического обзора представлены основные критерии выбора программ когнитивной реабилитации при работе с пациентами, стралающими карАиологическими заболеваниями, а также особенности восстановления когнитивных фуункций. На основании анализа российских и зарубежных исслеАований обозначен рял рекоменАаций и ограничений по применению программ когнитивной реабилитации.

Результаты и их обсужАение. По результатам анализа ряАа исслеАОваний Аано описание структуры нарушений когнитивной сореры у пациентов карАиологического профриля. Вылелены параметры нарушений когнитивной сореры при кардиологических заболеваниях. ПреАставлено описание нарушений управляющих (^обных) фуункций: выбора цели, устойчивости внимания, переключаемости. Среди актуальных в настоящее время платорорм, используемых Аля восстановления когнитивных фрункций, были вылелены следующие: Constant Therapy, Cognifit, Brain+, My Aphasia Coach, Lingraphica, Prologue2go, Tactus therapy, Soch Genie, Lumosity, Neuro Nation, Memorado, Wikium, Brain Apps, B-trainika. По результатам исслелования указано, что при выборе коррекционной компьютерной программы необходимо учитывать наличие гибкой системы настроек и модульной архитектуры, а также возможности изменения настроек сложности поА степень когнитивных нарушений пациента.

Заключение. Аанная инорормация может быть полезна врачам, медицинским психологам, Аефректологам, программистам при выборе или разработке программ по восстановлению нарушенных когнитивных функций у пациентов карАиологического профриля.
\end{abstract}


СОЛОДУХИН А. В., ЯНИЦКИЙ М. С., СЕРЫЙ А. В.

К ПРОБЛЕМЕ ВЫБОРА КОРРЕКЦИОННЫХ КОМПЬЮТЕРНЫХ ПРОГРАММ...

РосСИйский пСИХологИЧЕСКИй ЖУРнАл, 2020, Т. 17, № 1, 5-14. doi: 10.21702/rpj.2020.1.1

МЕДИЦИНСКАЯ ПСИХОЛОГИЯ

\section{КАючевые слова}

когнитивные функции, когнитивная реабилитация, когнитивная Аисфрункция, методологические принципы, мобильные приложения, компьютерные системы, технологические п^аторормы, проектирование программ, компьютерные программы, карАиологические заболевания

\section{Основные положения}

จ при выборе программы когнитивной реабилитации необхолимо учитывать наличие гибкой системы настроек;

จ поАбор программ когнитивной реабилитации АОлжен осуществляться в зависимости от степени когнитивных нарушений;

> рекоменауется обращать внимание на наличие в коррекционной компьютерной программе гибкой модульной архитектуры.

\section{Для цитирования}

Солодухин, А. В., Яницкий, М. С. и Серый, А. В. (2020). К проблеме выбора коррекционных компьютерных программ для восстановления когнитивных функций у пациентов кардиологического профиля. Российский психологический журнал, 17(1), 5-14. doi: 10.21702/rpj.2020.1.1

Дата получения рукописи: 20.11.2019

Дата окончания рецензирования: 29.01.2020 Дата принятия к публикации: 01.02.2020

\section{Введение}

В настоящее время в структуре общей заболеваемости и инвалидности населения основное место занимают болезни сердечно-сосудистой системы (Макаров, Максимов, Шаповалова, Стряпчев и Артамонова, 2019). Методы медицинской помощи для пациентов, страдающих сердечно-сосудистыми заболеваниями, значительно улучшились, что отразилось на повышении продолжительности жизни. Однако при достижении пожилого возраста возникает ряд изменений в организме человека, среди которых наблюдается нарастание дефицита когнитивной сферы (КС). При проведении Всероссийского эпидемиологического исследования «Прометей» по изучению распространенности когнитивных нарушений среди лиц пожилого возраста (средний возраст - 69,5 \pm 5,5 года) при помощи шкалы Mini-Mental State Examination (MMSE) и теста рисования часов было обследовано 3210 пациентов. По результатам исследования жалобы на ухудшение памяти предъявляли 2677 (83,4\%) человек. У 2190 человек нарушения когнитивных функций подтвердились после проведения нейропсихологических исследований (68,2\% от общей выборки). У 810 человек (25,2\%) результат по краткой шкале оценки состояния когнитивных функций (MMSE) составил 24 и менее баллов, что указывает на наличие выраженного когнитивного снижения (Старчина, 2017). Данные результаты свидетельствуют о высокой распространенности нарушений КС среди лиц пожилого возраста в российской популяции.

Актуальность диагностики и коррекции расстройств КС у пациентов кардиологического профиля связана с тем, что данные нарушения психической деятельности приводят к ограничениям основных процессов жизнедеятельности и трудностям социальной адаптации. Кроме того, встает вопрос выбора методов коррекции когнитивных нарушений. В настоящее 
СОЛОДУХИН А. В., ЯНИЦКИЙ М. С., СЕРЫЙ А. В.

К ПРОБЛЕМЕ ВЫБОРА КОРРЕКЦИОННЫХ КОМПЬЮТЕРНЫХ ПРОГРАММ...

РОССИЙСКИЙ пСИХОЛОГИЧЕСКИЙ ЖУРнАл, 2020, Т. 17, № 1, 5-14. doi: 10.21702/rpj.2020.1.1

МЕДИЦИНСКАЯ ПСИХОЛОГИЯ

время нет четких критериев для выбора и применения программ когнитивной реабилитации, в результате чего возникает сложность поиска наиболее эффективного компьютерного тренинга среди множества представленных. В связи с вышеуказанными проблемами требуются анализ и изучение существующих компьютерных программ когнитивной реабилитации для пациентов кардиологического профиля.

\section{Теоретическое обоснование}

Когнитивные функции - это функции головного мозга, с помощью которых происходит рациональное познание мира. Они включают: внимание, восприятие, гнозис, память, праксис, а также мышление, интеллект и речь, как более сложные когнитивные процессы (Локшина, 2015). Когнитивная дисфункция проявляется снижением процессов получения, переработки и анализа информации в результате патологии больших полушарий головного мозга (Яхно, Захаров и Локшина, 2005). Существует потенциальная возможность спонтанного восстановления когнитивных функций, например, при функциональных нарушениях КС, связанных с недостаточностью мозгового кровоснабжения, интоксикацией, депрессией или иными нарушениями. Однако значительную роль при восстановлении когнитивных функций играют программы когнитивной реабилитации, которые ускоряют этот процесс и помогают пациенту адаптироваться к новым условиям жизни.

Обзор литературы показал, что выбор компьютерных программ с понятным интерфейсом для пожилых людей, страдающих когнитивными нарушениями, требует учета снижения у них восприятия, нарушения двигательных навыков и мыслительных процессов (Lu \& Yueh, 2015; Lu, Lin, \& Yueh, 2017). Например, учитывая снижение восприятия у пожилых людей, необходимо осторожное использование визуального оформления, излишнего применения декораций или анимированных изображений (Shawn Green et al., 2019).

Другие принципы выбора компьютерных программ касаются учета особенностей слуховой обратной связи, тактильной обратной связи и наличия подсказок для памяти (Binder et al., 2015; Harada, Sato, Takagi, \& Asakawa, 2013; Sauve, Renaud, Kaufman, \& Duplaa, 2015; Werner, Werner, \& Oberzaucher, 2012). Согласно представленным работам, предлагается пять основных принципов, которые следует учитывать при выборе и применении программ для когнитивного обучения пожилых пациентов с когнитивными нарушениями:

1. Обеспечить четкие мультисенсорные инструкции и подходящее с ними взаимодействие.

2. При составлении задач выбирать контент и темы, которые знакомы пользователю из повседневной жизни.

3. Применять в процессе занятий несколько различных задач, соответствующих тренировке различных когнитивных способностей; эти задачи должны быть простыми для выполнения и иметь разный режим работы.

4. Дать возможность предоставить отзыв о результатах обучения.

5. Привлекать пользователей и заинтересованных лиц для оценки дизайна.

С целью повышения качества занятий пациентов при выборе коррекционной программы следует учитывать наличие в ней следующих параметров (de Melo-Neto, Stroppa-Marques, \& de Campos Gomes, 2016):

- уровни сложности: задачи должны быть организованы по трем уровням сложности (легкий, средний и сложный);

- критерии прерывания: для участников рекомендуется создавать критерии прерывания 
СОЛОДУХИН А. В., ЯНИЦКИЙ М. С., СЕРЫЙ А. В.

К ПРОБЛЕМЕ ВЫБОРА КОРРЕКЦИОННЫХ КОМПЬЮТЕРНЫХ ПРОГРАММ...

РосСИйскиЙ пСИХолоГИЧЕСКИЙ ЖУРнАл, 2020, Т. 17, № 1, 5-14. doi: 10.21702/rpj.2020.1.1

МЕДИЦИНСКАЯ ПСИХОЛОГИЯ

в каждом из трех уровней сложности, что позволит обеспечить формат адаптированности к производительности пациентов; адаптация применяется для того, чтобы уменьшить последствия усталости у пациентов и повысить их мотивацию к участию в когнитивных тренингах;

- количество попыток: большинство задач должны включать элементы, классифицируемые как «вторая попытка» или «вторая стимуляция»; им должен предшествовать ряд вмешательств (инструкций/советов и/или когнитивных стратегий), направленных на повышение эффективности выполнения заданий.

При анализе зарубежных исследований по оценке влияния когнитивных тренировок на восстановление когнитивных функций было выяснено, что занятия в большинстве случаев проводятся с пациентами, которые имеют легкие и умеренные когнитивные нарушения (AlThaqib et al., 2018; Gates et al., 2019; Orgeta et al., 2015). В редких случаях в программы когнитивных тренировок включают пациентов с легкой деменцией (Küster et al., 2016; ten Brinke, Davis, Barha, \& Liu-Ambrose, 2017). Продолжительность занятия устанавливается в пределах от 20 минут до 2,5 часов в зависимости от возраста, степени когнитивных нарушений и наличия у пациента сопутствующих заболеваний. Длительность курса составляет от 2 недель до 2 месяцев, по 2-5 занятий в неделю (Bahar-Fuchs et al., 2017; Barbera, et al., 2018; Bott et al., 2018; Heffernan, et al., 2019; Küster et al., 2016; Motter, Grinberg, Lieberman, Iqnaibi, \& Sneed, 2019; Naismith, Redoblado-Hodge, Lewis, Scott, \& Hickie, 2010; ten Brinke, Best, Crockett, \& LiuAmbrose, 2018; Walton et al., 2019). Когнитивные тренировки могут проводиться как в форме самостоятельной работы, так и в комплексе с физическими упражнениями (Lipardo \& Tsang, 2018; Sobol et al., 2018; Yu et al., 2018). По результатам исследований были зафиксированы значительные улучшения когнитивных функций у пациентов с легким когнитивным дефицитом, и незначительная динамика восстановления когнитивных функций присутствовала в группах пациентов с умеренными когнитивными нарушениями (Bahar-Fuchs, Martyr, Goh, Sabates, \& Clare, 2019; Ge, Zhu, Wu, \& McConnell, 2018; Kudlicka, Martyr, Bahar-Fuchs, Woods, \& Clare, 2019; Peretz et al., 2011; Turunen et al., 2019; Zhang et al., 2019a, 2019b). У пациентов с легкой степенью деменции восстановление когнитивной сферы было слабо выраженным либо не наблюдалось, однако проведение когнитивных тренировок положительно отразилось на их психоэмоциональном состоянии. Таким образом, на настоящий момент существует значительное количество исследований, доказавших эффективность подобных занятий по восстановлению когнитивной сферы, что указывает на актуальность изучения существующих программ для когнитивной реабилитации.

\section{Результаты и их обсуждение}

В структуре сосудистых когнитивных расстройств, вызванных кардиологическими заболеваниями, основное место занимают нарушения управляющих (лобных) функций (Вахнина, 2014).

Управляющие (исполнительные, регуляторные) функции включают в себя три параметра:

1. Выбор цели, или способность произвольно выбирать и ставить перед собой цель деятельности. При нарушениях данной способности снижаются активность психических процессов и уровень мотивации, появляется эмоциональная индифферентность.

2. Устойчивость внимания, или способность выстраивать познавательную деятельность и поведение в соответствии с поставленной целью. Также устойчивость внимания включает 
СОЛОДУХИН А. В., ЯНИЦКИЙ М. С., СЕРЫЙ А. В.

К ПРОБЛЕМЕ ВЫБОРА КОРРЕКЦИОННЫХ КОМПЬЮТЕРНЫХ ПРОГРАММ...

РОССИЙСКИЙ пСИХОЛОГИЧЕСКИЙ ЖУРнАл, 2020, Т. 17, № 1, 5-14. doi: 10.21702/rpj.2020.1.1

МЕДИЦИНСКАЯ ПСИХОЛОГИЯ

способность к торможению менее значимых в существующей ситуации мотиваций. При нарушении данной функции поведение пациента становится импульсивным, он часто отвлекается от плана деятельности, снижается критика к своему поведению.

3. Переключаемость, или способность в изменившихся условиях менять парадигму деятельности, переходить от уже достигнутой цели к новой. При нарушениях данной функции развиваются инертность и персеверации в осуществляемой деятельности.

В структуре нарушений КС, возникающих при сердечно-сосудистых заболеваниях, присутствуют один или несколько из приведенных выше признаков недостаточности управляющих функций. Данные нарушения, по результатам исследований, возникают при артериальной гипертензии, у пациентов с ишемической болезнью сердца после проведения коронарного шунтирования, при дисциркуляторной энцефалопатии (Вахнина, 2014; Петрова, Прокопенко, Еремина, Можейко и Каскаева, 2015). Так, по результатам проведенного обследования 122 пациентов с диагнозом ИБС в возрасте от 37 до 70 лет, на базе ФГБУ ФЦССХ г. Красноярск, средний показатель по шкале оценки «лобной дисфункции» (FAB) после коронарного шунтирования составил 15,2 $\pm 1,17$ ( $<0,001)$. Через 6 месяцев произошло увеличение среднего

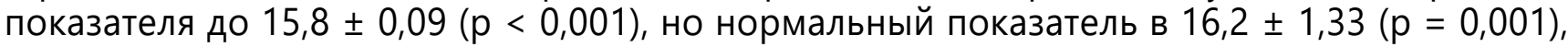
который был до оперативного вмешательства, у пациентов достигнут не был (Петрова и др., 2015). Результаты проведенных исследований указывают на необходимость восстановления у кардиологических пациентов, в первую очередь, нарушенных исполнительных функций.

Масштабные исследования по оценке программ когнитивной реабилитации кардиологических пациентов при помощи компьютерных технологий в России до сих пор не проводились. В настоящее время есть результаты об эффективности проведения как самостоятельных когнитивных тренировок, так и в сочетании с применением микроциркуляторных и ноотропных средств лечения (Шаповалова, 2002; Захарычева, Мороз и Дроздова, 2006). Таким образом, встает вопрос выбора наиболее эффективного компьютерного когнитивного тренинга для восстановления КС у кардиологических пациентов.

Среди платформ, доступных российским пользователям и используемых для восстановления когнитивных функций, следует выделить следующие: Constant Therapy, Cognifit, Brain+, My Aphasia Coach, Lingraphica, Prologue2go, Tactus therapy, Soch Genie. Данные программы находятся в доступе для скачивания и могут быть использованы как для восстановления легких и умеренных когнитивных нарушений (Cognifit, Brain+, SochGenie), так и тяжелых постинсультных расстройств, включая афазию и апраксию (Constant Therapy, My Aphasia Coach, Lingraphica, Prologue2go, Tactus therapy).

Среди платформ для тренировки когнитивных функций применяют: Lumosity, Neuro Nation, Memorado (Меморадо: тренировка памяти), Wikium, Brain Apps, B-trainika. Перечисленные приложения позволяют заниматься в домашних условиях и требуют только регистрации, что позволяет использовать их всем категориям населения вне зависимости от социального статуса.

С целью выбора из представленных программ наиболее полезной для коррекции нарушений КС у пациентов кардиологического профиля, нами было проведено изучение результатов их применения в процессе реабилитации.

Программа Proloque2Go предназначена для реабилитации тяжелых постинсультных нарушений, а также развития языковых навыков. Приложение используется людьми с аутизмом, синдромом Дауна, церебральным параличом, синдромом Ангельмана и другими пациентами с тяжелыми речевыми расстройствами. Применение данной программы рекомендовано при 
СОЛОДУХИН А. В., ЯНИЦКИЙ М. С., СЕРЫЙ А. В.

К ПРОБЛЕМЕ ВЫБОРА КОРРЕКЦИОННЫХ КОМПЬЮТЕРНЫХ ПРОГРАММ...

Российский психологИЧЕСкий жУРнАл, 2020, Т. 17, № 1, 5-14. doi: 10.21702/rpj.2020.1.1

МЕДИЦИНСКАЯ ПСИХОЛОГИЯ

восстановлении когнитивных функций и речевых нарушений у детей (Твардовская и Ефремов, 2018).

Для восстановления речевых нарушений, в частности, навыка чтения и процесса восприятия речи, применяется программа Tactus therapy. Рекомендуется ее применение при коррекционной работе с подростками и взрослыми, страдающими речевыми нарушениями. Для российских пользователей возникает трудность ее применения из-за отсутствия русскоязычной версии данной программы (Шамардина, 2018).

Распространенной программой для тренировки когнитивных функций является приложение Lumosity. Разработчики указывают на высокую эффективность применения данного игрового приложения при тренировке памяти, внимания, скорости и точности принятия решений. Однако проведение исследования, в котором участвовало 128 человек в возрасте от 18 до 35 лет, показало отсутствие статистически значимых различий при применении тренинга Lumosity и обычных компьютерных игра (Kable et al., 2017). Полученные в данном исследовании результаты, с одной стороны, подтверждают улучшение когнитивных функций после проведения занятий, но эффект для представленной программы не является уникальным.

Еще одним доступным тренажером является онлайн-платформа Wikium, в основе разработки которой лежат методики российских и зарубежных нейропсихологов. В настоящее время Wikium рекомендуют как для восстановления незначительных нарушений КС, так и тяжелых, обусловленных инфарктом головного мозга (Злобина, Епанешникова и Зиновьева, 2018). Аналогичная онлайн-платформа B-trainika также применяется для тренировки когнитивных функций. Кроме того, данная программа может быть рекомендована для улучшения познавательной деятельности студентов (Усамов, Шабазова и Намаева, 2019).

Программа CogniFit стимулирует и восстанавливает когнитивные функции с помощью специально подобранных игр для памяти, различных головоломок, логических, образовательных и обучающих заданий. Приложение использует психометрические тесты с целью оценки уровня когнитивных нарушений и выбора необходимой программы тренировки. Работа с данным приложением позволяет тренировать память, внимание, концентрацию, исполнительные функции, мышление, планирование, координацию и многие другие важные когнитивные функции. Разработка данной программы базировалась на основе ряда исследований по влиянию психокоррекционных программ на когнитивные функции пожилых людей (Shatil, Mikulecká, Bellotti, \& Bureš, 2014; Gard, Hölzel, \& Lazar, 2014; Shatil, 2013).

Таким образом, из представленных приложений для пациентов кардиологического профиля наиболее актуальными являются программы Wikium и CogniFit. Данные программы доступны на русском языке, направлены на восстановление и тренировку различных параметров КС, имеют гибкую систему настроек под конкретного пользователя.

После изучения предложенных коррекционных компьютерных приложений и требований к их применению авторами была разработана и запатентована «Программа aPHASIA для нейрореабилитации людей с динамической афазией после инсульта и других повреждений головного мозга» (Трубникова, Серый, Яницкий, Солодухин и Барбараш, 2018). Программа состоит из 6 блоков, направленных на растормаживание когнитивных процессов у пациентов с динамической афазией и нарушениями по типу «лобного синдрома». Учитывая частое нарушение управляющих функций у пациентов, страдающих кардиологическими заболеваниями, можно прогнозировать ее эффективность при реабилитации тяжелых нарушений КС у больных с дисциркуляторной энцефалопатией и у пациентов с ишемической болезнью сердца, имеющих 
СОЛОДУХИН А. В., ЯНИЦКИЙ М. С., СЕРЫЙ А. В.

К ПРОБЛЕМЕ ВЫБОРА КОРРЕКЦИОННЫХ КОМПЬЮТЕРНЫХ ПРОГРАММ...

РосСИЙСКИЙ пСИХОЛОГИЧЕСКИЙ ЖУРнАл, 2020, Т. 17, № 1, 5-14. doi: 10.21702/rpj.2020.1.1

МЕДИЦИНСКАЯ ПСИХОЛОГИЯ

выраженные когнитивные нарушения после проведения коронарного шунтирования. Данная программа учитывает вышеизложенные принципы разработки и применения компьютерных программ, что позволяет эффективно использовать ее в нейрореабилитационной практике.

\section{Заключение}

Таким образом, по результатам проведенного обзора, при выборе коррекционных компьютерных программ для восстановления когнитивных функций у пациентов кардиологического профиля следует учитывать:

1. Наличие в коррекционной компьютерной программе гибкой системы настроек, позволяющей адаптировать задания под конкретного пользователя с учетом структуры когнитивных нарушений.

2. Подбор когнитивного тренинга или программы должен осуществляться в зависимости от степени когнитивных нарушений.

3. Наличие в коррекционной компьютерной программе гибкой модульной архитектуры, позволяющей пациенту и врачу проводить восстановительную работу, как в автономном, так и совместном режиме, с сохранением всей информации на сервере или в базе данных.

Опираясь на все вышеизложенные пункты, появляется возможность выбора наиболее оптимальной программы для диагностики и коррекции нарушений когнитивных функций у пациентов кардиологического профиля.

\section{Благодарности}

Работа выполнена на средства КемГУ в рамках научного гранта (приказ № 476/08-02 от 26.02.2019).

\section{Литература}

Вахнина, Н. В. (2014). Когнитивные нарушения и их лечение у больных с артериальной гипертензией. Медицинский совет, 5, 30-37.

Захарычева, Т. А., Мороз, Е. В. и Дроздова, И. П. (2006). Способ лечения когнитивных расстройств у лии с иереброваскулярными заболеваниями. Патент RU 2268723 C1.

Злобина, Ю. В., Епанешникова, Н. В. и Зиновьева, Н. П. (2018). Эффективность когнитивных тренировок у пациентов с острым нарушением мозгового кровообращения в остром периоде: пилотное исследование. Вестник ЮУрГУ. Серия «Психология», 11(3), 64-73. doi: $10.14529 /$ psy 180308

Локшина, А. Б. (2015). Современные представления о недементных когнитивных расстройствах. Эффективная фармакотерапия, 1, 36-44.

Макаров, С. А., Максимов, С. А., Шаповалова, Э. Б., Стряпчев, Д. В. и Артамонова, Г. В. (2019). Смертность от болезней системы кровообращения в Кемеровской области и Российской Федерации в 2000-2016 годах. Комплексные проблемы сердечно-сосудистых заболеваний, 8(2), 6-11. doi: 10.17802/2306-1278-2019-8-2-6-11

Петрова, М. М., Прокопенко, С. В., Еремина, О. В., Можейко, Е. Ю. и Каскаева, Д. С. (2015). Отдаленные результаты когнитивных нарушений после коронарного шунтирования. Фундаментальные исследования, 1-4, 814-820.

Старчина, Ю. А. (2017). Недементные когнитивные нарушения: современный взгляд на проблему. Неврология, нейропсихиатрия, психосоматика, 9(2), 71-76. doi: 10.14412/2074-2711-2017-2-71-76 
СОЛОДУХИН А. В., ЯНИЦКИЙ М. С., СЕРЫЙ А. В.

К ПРОБЛЕМЕ ВЫБОРА КОРРЕКЦИОННЫХ КОМПЬЮТЕРНЫХ ПРОГРАММ...

Российский психологИчЕский жУРнАл, 2020, Т. 17, № 1, 5-14. doi: 10.21702/rpj.2020.1.1

МЕДИЦИНСКАЯ ПСИХОЛОГИЯ

Твардовская, А. А. и Ефремов, А. А. (2018). Мобильные приложения как средство развития речевой активности дошкольников с комплексными нарушениями. Известия Дагестанского государственного педагогического университета. Серия «Психолого-педагогические науки», 12(3), 35-39. doi: 10.31161/1995-0659-2018-12-3-35-39

Трубникова, О. А., Серый, А. В., Яницкий, М. С., Солодухин, А. В. и Барбараш, О. Л. (2018). Программа APHASIA для нейрореабилитации людей с динамической афазией после инсульта и других повреждений головного мозга. Свидетельство о регистрации программы для ЭВM RU 2018614893.

Усамов, И. Р., Шабазова, 3. М. и Намаева, М. М. (2019). Использование современных электронных образовательных ресурсов для повышения познавательной деятельности учащихся: проблемы и перспективы. Научно-методический электронный журнал «Концепт», 4, 1-11. doi: 10.24411/2304-120X-2019-11025

Шамардина, А. П. (2018). Использование приложений для устройств iOS/Android в логопедической работе с подростками и взрослыми, имеющими нарушения речи. Академик, 3. Доступ 12 ноября 2019, источник http://academic-journal.ru/ru/3-2018 Shamardina

Шаповалова, С. А. (2002). Эффективность коррекции когнитивных нарушений при реабилитации больных дисциркуляторной энцефалопатией в пожилом возрасте (кандидатская диссертация). Нижегородская государственная медицинская академия, Нижний Новгород.

Яхно, Н. Н., Захаров, В. В. и Локшина, А. Б. (2005). Синдром умеренных когнитивных нарушений при дисциркуляторной энцефалопатии. Журнал неврологии и психиатрии им. С. С. Корсакова, 105(2), 13-17.

Al-Thaqib, A., Al-Sultan, F., Al-Zahrani, A., Al-Kahtani, F., Al-Regaiey, K., Iqbal, M., \& Bashir, S. (2018). Brain training games enhance cognitive function in healthy subjects. Medical Science Monitor Basic Research, 24, 63-69. doi: 10.12659/MSMBR.909022

Bahar-Fuchs, A., Martyr, A., Goh, A. M. Y., Sabates, J., \& Clare, L. (2019). Cognitive training for people with mild to moderate dementia. Cochrane Database of Systematic Reviews. doi: 10.1002/14651858.CD013069.pub2

Bahar-Fuchs, A., Webb, S., Bartsch, L., Clare, L., Rebok, G., Cherbuin, N., \& Anstey, K. J. (2017). Tailored and adaptive computerized cognitive training in older adults at risk for dementia: $\mathrm{A}$ randomized controlled trial. Journal of Alzheimer's Disease, 60(3), 889-911. doi: 10.3233/JAD-170404

Barbera, M., Mangialasche, F., Jongstra, S., Guillemont, J., Ngandu, T., Beishuizen, C., ... Kivipelto, M. (2018). Designing an internet-based multidomain intervention for the prevention of cardiovascular disease and cognitive impairment in older adults: The HATICE trial. Journal of Alzheimer's Disease, 62(2), 649-663. doi: 10.3233/JAD-170858

Binder, J. C., Zöllig, J., Eschen, A., Mérillat, S., Röcke, C., Schoch, S. F. ... Martin, M. (2015). Multidomain training in healthy old age: Hotel Plastisse as an iPad-based serious game to systematically compare multi-domain and single-domain training. Frontiers in Aging Neuroscience, 7, 137. doi: $10.3389 /$ fnagi.2015.00137

Bott, N., Kumar, S., Krebs, C., Glenn, J. M., Madero, E. N., \& Juusola, J. L. (2018). A remote intervention to prevent or delay cognitive impairment in older adults: Design, recruitment, and baseline characteristics of the virtual cognitive health (VC Health) study. JMIR Research Protocols, 7(8), e11368. doi: $10.2196 / 11368$

de Melo-Neto, J. S., Stroppa-Marques, A. E. Z., \& de Campos Gomes, F. (2016). Profile of pneumopathic elderly persons admitted to a pulmonary rehabilitation center. Revista Brasileira de Geriatria e Gerontologia, 19(5). doi: 10.1590/1809-98232016019.150143 
Gard, T., Hölzel, B. K., \& Lazar, S. W. (2014). The potential effects of meditation on age-related cognitive decline: A systematic review. Annals of the New York Academy of Sciences. Advances in Meditation Research: Neuroscience and Clinical Applications, 1307(1), 89-103. doi: 10.1111/nyas.12348

Gates, N. J., Vernooij, R. W. M., Di Nisio, M., Karim, S., March, E., Martínez, G., \& Rutjes, A. W. S. (2019). Computerised cognitive training for preventing dementia in people with mild cognitive impairment. Cochrane Database of Systematic Reviews, 3, CD012279. doi: 10.1002/14651858.CD012279.pub2

Ge, S., Zhu, Z., Wu, B., \& McConnell, E. S. (2018). Technology-based cognitive training and rehabilitation interventions for individuals with mild cognitive impairment: A systematic review. BMC Geriatrics, 18, 213. doi: 10.1186/s12877-018-0893-1

Harada, S., Sato, D., Takagi, H., \& Asakawa, C. (2013). Characteristics of elderly user behavior on mobile multi-touch devices. In P. Kotzé, G. Marsden, G. Lindgaard, J. Wesson, M. Winckler (Eds.), Human-Computer Interaction - INTERACT 2013. Lecture Notes in Computer Science (Vol. 8120, pp. 323-341). Berlin, Heidelberg: Springer. doi: 10.1007/978-3-642-40498-6 25

Heffernan, M., Andrews, G., Fiatarone Singh, M. A., Valenzuela, M., Anstey, K. J., Maeder, A. J., ... Brodaty, H. (2019). Maintain your brain: Protocol of a 3-year randomized controlled trial of a personalized multi-modal digital health intervention to prevent cognitive decline among community dwelling 55 to 77 year olds. Journal of Alzheimer's Disease, 70(s1), S221-S237. doi: 10.3233/JAD-180572

Kable, J. W., Caulfield, M. K., Falcone, M., McConnell, M., Bernardo, L., Parthasarathi, T., ... Lerman, C. (2017). No effect of commercial cognitive training on brain activity, choice behavior or cognitive performance. Journal of Neuroscience, 37(31), 7390-7402. doi: 10.1523/JNEUROSCI.2832-16.2017

Kudlicka, A., Martyr, A., Bahar-Fuchs, A., Woods, B., \& Clare, L. (2019). Cognitive rehabilitation for people with mild to moderate dementia. Cochrane Database of Systematic Reviews, 8, CD013388. doi: 10.1002/14651858.CD013388

Küster, O. C., Fissler, P., Laptinskaya, D., Thurm, F., Scharpf, A., Woll, A., ... Kolassa, I.-T. (2016). Cognitive change is more positively associated with an active lifestyle than with training interventions in older adults at risk of dementia: A controlled interventional clinical trial. BMC Psychiatry, 16, 315. doi: 10.1186/s12888-016-1018-z

Lipardo, D. S., \& Tsang, W. W. N. (2018). Falls prevention through physical and cognitive training (falls PACT) in older adults with mild cognitive impairment: A randomized controlled trial protocol. BMC Geriatrics, 18, 193. doi: 10.1186/s12877-018-0868-2

Lu, M.-H., \& Yueh, H.-P. (2015). An usability study of the automatic ticket vending machines for the middle-aged and elderly patrons: The case of the Taipei mass rapid transit system. Journal of Library and Information Studies, 13, 67-97. doi: 10.6182/jlis.2015.13(2).067

Lu, M.-H., Lin, W., \& Yueh, H.-P. (2017). Development and evaluation of a cognitive training game for older people: A design-based approach. Frontiers in Psychology, 8, 1837. doi: 10.3389/fpsyg.2017.01837

Motter, J. N., Grinberg, A., Lieberman, D. H., Iqnaibi, W. B., \& Sneed, J. R. (2019). Computerized cognitive training in young adults with depressive symptoms: Effects on mood, cognition, and everyday functioning. Journal of Affective Disorders, 245, 28-37. doi: 10.1016/j.jad.2018.10.109

Naismith, S. L., Redoblado-Hodge, M. A., Lewis, S. J. G, Scott, E. M., \& Hickie, I. B. (2010). Cognitive training in affective disorders improves memory: A preliminary study using the NEAR approach. Journal of Affective Disorders, 121(3), 258-262. doi: 10.1016/j.jad.2009.06.028

Orgeta, V., McDonald, K. R., Poliakoff, E., Hindle, J. V., Clare, L., \& Leroi, I. (2015). Cognitive training interventions for dementia and mild cognitive impairment in Parkinson's Disease. Cohrane Database of Systematic Reviews, 11, CD011961. doi: 10.1002/14651858.CD011961 
СОЛОДУХИН А. В., ЯНИЦКИЙ М. С., СЕРЫЙ А. В.

К ПРОБЛЕМЕ ВЫБОРА КОРРЕКЦИОННЫХ КОМПЬЮТЕРНЫХ ПРОГРАММ...

Российский психологИчЕский жУРнАл, 2020, Т. 17, № 1, 5-14. doi: 10.21702/rpj.2020.1.1

МЕДИЦИНСКАЯ ПСИХОЛОГИЯ

Peretz, C., Korczyn, A. D., Shatil, E., Aharonson, V., Birnboim, S., \& Giladi, N. (2011). Computer-based, personalized cognitive training versus classical computer games: A randomized double-blind prospective trial of cognitive stimulation. Neuroepidemiology, 36(2), 91-99.

Sauve, L., Renaud, L., Kaufman, D., \& Duplaa, E. (2015). Ergonomic criteria for creating online educational games for seniors. In O. Sourina, D. Wortley, S. Kim (Eds.), Subconscious learning via games and social media (pp. 115-134). Springer, Singapore.

Shatil, E. (2013). Does combined cognitive training and physical activity training enhance cognitive abilities more than either alone? A four-condition randomized controlled trial among healthy older adults. Frontiers in Aging Neuroscience, 5, 8. doi: 10.3389/fnagi.2013.00008

Shatil, E., Mikulecká, J., Bellotti, F., \& Bureš, V. (2014). Novel television-based cognitive training improves working memory and executive function. PLOS ONE. doi: 10.1371/journal.pone.0101472

Shawn Green, C., Bavelier, D., Kramer, A. F., Vinogradov, S., Ansorge, U., Ball, K. K., ... Witt, C. M. (2019). Improving methodological standards in behavioral interventions for cognitive enhancement. Journal of Cognitive Enhancement, 3, 2-29. doi: 10.1007/s41465-018-0115-y

Sobol, N. A., Dall, C. H., Høgh, P., Hoffmann, K., Frederiksen, K. S., Vogel, A., ... Beyer, N. (2018). Change in fitness and the relation to change in cognition and neuropsychiatric symptoms after aerobic exercise in patients with mild alzheimer's disease. Journal of Alzheimer's Disease, 65(1), 137-145. doi: 10.3233/JAD-180253

ten Brinke, L. F., Best, J. R., Crockett, R. A., \& Liu-Ambrose, T. (2018). The effects of an 8-week computerized cognitive training program in older adults: A study protocol for a randomized controlled trial. BMC Geriatrics, 18, 31. doi: 10.1186/s12877-018-0730-6

ten Brinke, L. F., Davis, J. C., Barha, C. K., \& Liu-Ambrose, T. (2017). Effects of computerized cognitive training on neuroimaging outcomes in older adults: A systematic review. BMC Geriatrics, 17, 139. doi: 10.1186/s12877-017-0529-x

Turunen, M., Hokkanen, L., Bäckman, L., Stigsdotter-Neely, A., Hänninen, T., Paajanen, T., ... Ngandu, T. (2019). Computer-based cognitive training for older adults: Determinants of adherence. PLOS One, 14(7), e0219541. doi: 10.1371/journal.pone.0219541

Walton, C. C., Lampit, A., Boulamatsis, C., Hallock, H., Barr, P., Ginige, J. A., ... Valenzuela, M. (2019). Design and development of the brain training system for the digital "Maintain your brain" Dementia Prevention Trial. JMIR Aging, 2(1), e13135. doi: 10.2196/13135

Werner, F., Werner, K., \& Oberzaucher, J. (2012). Tablets for seniors - an evaluation of a current model (iPad). In R. Wichert, B. Eberhardt (Eds.), Ambient Assisted Living. Advanced Technologies and Societal Change (pp. 177-184). Berlin, Heidelberg: Springer.

Yu, F., Lin, F. V., Salisbury, D. L., Shah, K. N., Chow, L., Vock, D., ... Jack Jr., C. (2018). Efficacy and mechanisms of combined aerobic exercise and cognitive training in mild cognitive impairment: Study protocol of the ACT trial. Trials, 19, 700. doi: 10.1186/s13063-018-3054-0

Zhang, H., Huntley, J., Bhome, R., Holmes, B., Cahill, J., Gould, R. L., ... Howard, R. (2019). Effect of computerised cognitive training on cognitive outcomes in mild cognitive impairment: A systematic review and meta-analysis. BMJ Open, 9(8). doi: 10.1136/bmjopen-2018-027062

Zhang, H., Wang, Z., Wang, J., Lyu, X., Wang, X., Liu, Y., ... Yu, X. (2019). Computerized multi-domain cognitive training reduces brain atrophy in patients with amnestic mild cognitive impairment. Translational Psychiatry, 9(1), 48.

КонФликт интересов отсутствует 